\title{
Meaning Generation for Animals, Humans and Artificial Agents. An Evolutionary Perspective on the Philosophy of Information ${ }^{\dagger}$
}

\author{
Christophe Menant \\ Independant scholar, Bordeaux, France; christophe.menant@hotmail.fr; Tel.: +33-061-021-2106 \\ + Presented at the IS4SI 2017 Summit DIGITALISATION FOR A SUSTAINABLE SOCIETY, Gothenburg, \\ Sweden, 12-16 June 2017.
}

Published: 8 June 2017

\begin{abstract}
Meanings are present everywhere in our environment and within ourselves. But these meanings do not exist by themselves. They are associated to information and have to be created, to be generated by agents. The Meaning Generator System (MGS) has been developed to model meaning generation in agents following a system approach in an evolutionary perspective. The agents can be natural or artificial. The MGS generates meaningful information (a meaning) when it receives information that has a connection with an internal constraint to which the agent is submitted. The generated meaning is to be used by the agent to implement actions aimed at satisfying the constraint. We propose here to highlight some characteristics of the MGS that could be related to items of philosophy of information.
\end{abstract}

Keywords: information; meaning; constraint; representation; evolution; self-consciousness; anxiety management; philosophy of information; ethics; symbol grounding problem

\section{Introduction}

Meanings are present everywhere. These meanings are associated to information and have to be generated by agents. We humans manage meanings permanently, consciously or not. We are meaning generating agents. Animals also are (a mouse seeing a cat will generate a meaning: 'danger'). Such a perspective can be extended to Artificial Agents (AAs) where the detection of an obstacle can generate a meaning within a robot programed to avoid obstacles. A system approach to meaning generation based on internal constraint satisfaction has been developed in an evolutionary perspective for animals, humans and AAs (the Meaning Generator System) [1]. We look here at positioning the MGS relatively to elements of the philosophy of information. We begin by presenting the MGS as being a system that generates meaningful information when it receives information that has a connection with the constraint. The generated meaning is to be used by the agent containing he MGS for action implementation aimed at constraint satisfaction. We highlight the characteristics of the MGS in order to see how some could be linked to philosophy of information [2].

Following an evolutionary approach we begin by addressing meaning generation in animal life where agents are submitted to 'stay alive' and 'live group life' constraints. Such simple cases can be modeled with a meaning generation structure that brings up the MGS.

The MGS is next extended to humans with challenges related to our limited understanding of human mind where new conscious and unconscious constraints drive the meaning generation processes. To get some understanding of human constraints we use an evolutionary scenario for self-consciousness based on the evolution of meaningful representations.

Meaning generation in AAs is the next step. Constraints in AAs are different from the ones existing in living entities. The latter are intrinsic to the agent but the former are derived from the human designer of the AA. These derived constraints participate to the generation of derived 
meaning. Using the MGS for AAs brings up subjects related to the meaning of information in Artificial Intelligence (AI) [3].

Highlighting the characteristics of the MGS for these different usages allows to compare them to some aspects of Philosophy of Information (PI).

\section{Meaning Generation in Animals, Humans and Artificial Agents. The Meaning Generator System}

The Meaning Generator System is represented in Figure 1. It is part of an agent submitted to an internal constraint. The generated meaning is information leading the agent to implement an action satisfying the constraint in a dynamic mode. The action can be internal or external to the agent, and it can be physical, biological or mental.

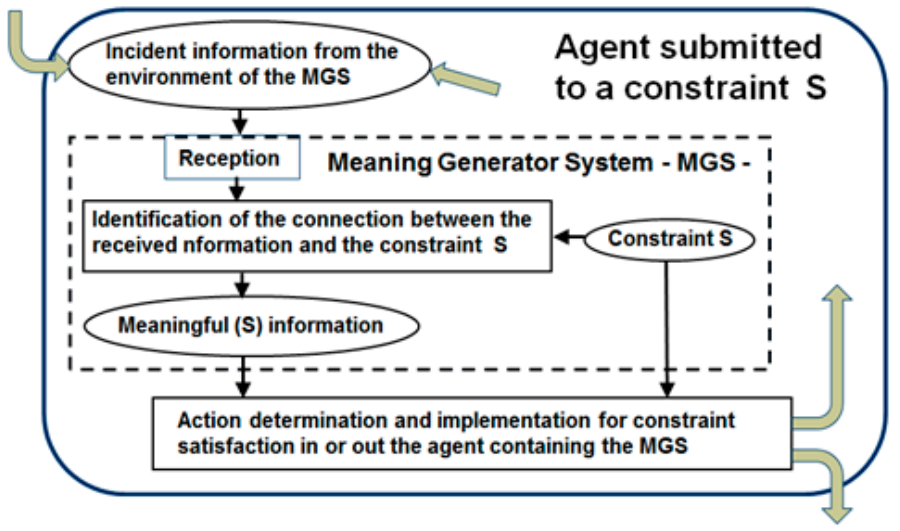

\section{MGS: System approach}

Animal constraints:

(Intrinsic)

- Stay alive (individual \& species)

- Live group life

Human constraints:

(Intrinsic)

- Limit anxiety

- Look for happiness

- Valorize ego,...

Artificial Agent constraints:

(Derived)

- As programmed

Figure 1. The Meaning Generator System (MGS).

The MGS is usable for basic life: the meaning 'danger' generated in a mouse submitted to a 'stay alive' constraint will trigger a hide or run away action to satisfy the constraint. The stay alive constraint applies to individual and to species, and both can be conflicting (ants can get drowned to be a bridge for other ants).

As said, application to humans is more complex as the understanding of human constraints is limited by our lack of visibility on the nature of human mind. To reach some understanding of human constraints we use an evolutionary scenario for self-consciousness that introduces anxiety limitation as a generic human constraint [4]. The scenario is based on the evolution of meaningful representations. It describes what could have been an evolution toward an elementary form of self-consciousness (an 'ancestral self-consciousness') resulting from pre-human primates becoming able to represent their own entities as existing in the environment like conspecifics were represented. Such performance is assumed as having been possible by an evolution of inter-subjectivity into identification with conspecifics at pre-human times. But the identification with conspecifics was also about identifying with suffering or endangered conspecifics that has produced a huge anxiety increase within our ancestors, an 'ancestral anxiety', having the same evolutionary origin that our ancestral self-consciousness. Anxiety limitation processes implemented to limit that anxiety increase have procured key evolutionary advantages and structured an 'evolutionary engine' favoring the evolution toward humans. That evolutionary scenario links the nature of human consciousness to anxiety management.

The evolutionary scenario is summarized in Figure 2. 


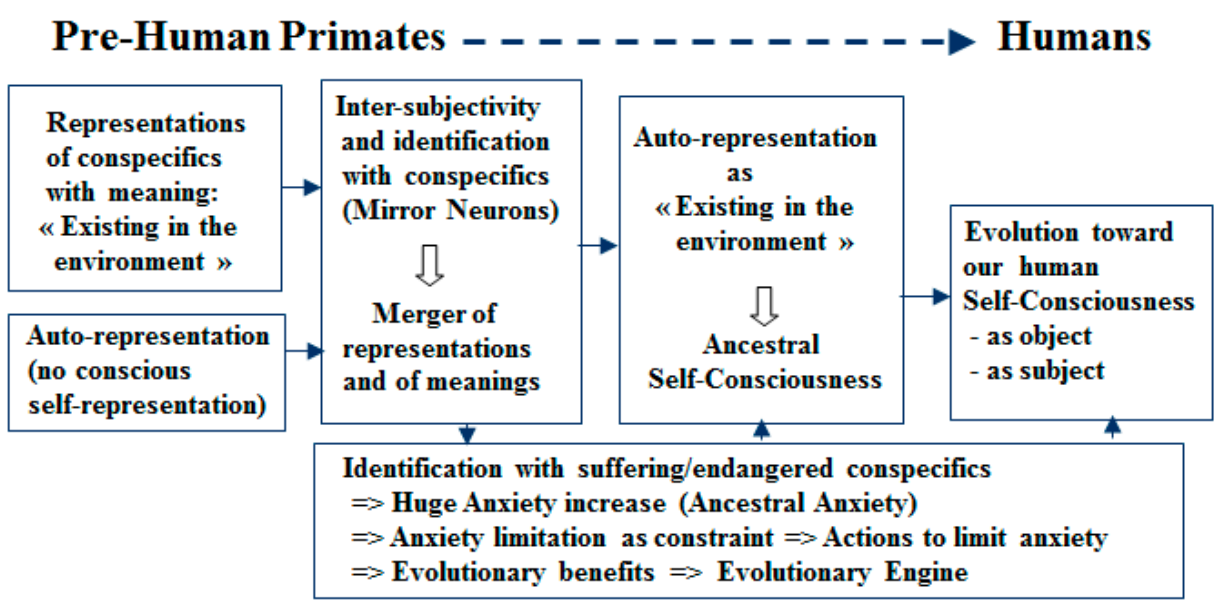

Figure 2. Meaning generation and self-consciousness. (Short version of https://philpapers.org/rec/ MENPFA-3).

As introduced above, using the MGS for Artificial Agents (AAs) brings in constraints of a different type than the natural/intrinsic ones existing in living entities. The internal constraints of AAs come from their designer, they are derived constraints that produce derived meanings. Such characterization of AAs introduces a system approach on the Symbol Grounding Problem (SGP) and to ethical concerns related to AI [3].

\section{Philosophy of Information and the MGS}

The philosophy of information (PI) is a huge research domain [5]. The focus of PI on information brings to look at possible compatibilities with the MGS approach. Among the many possible items to be compared we propose to look at the following ones:

- Meaningfulness. The MGS considers meaningful and meaningless information. PI deals with semantic information as well formed, meaningful and truthful data [5].

- Meaning generation. The function of meaning generation is at the core of the MGS approach. PI does not prioritize that aspect in semantic information.

- Evolution. The MGS is an evolutionary approach to meaning generation. Current PI does not explicitly consider evolution as an active part of semantic information.

- Veridicality thesis. PI considers that semantic information has to encapsulate truthfulness. The MGS does not use truth as a component of meaningful information.

- Symbol grounding problem. MGS and PI both address the SGP but they reach different conclusions $[3,6,7]$.

- Ontological status of information. PI addresses that question in terms of ontological category. MGS proposes an option with potential local constraints in a pre-biotic world.

- Naturalization of meaning. PI addresses this subject under various perspectives. MGS proposes an answer based on internal constraint satisfaction [5,8].

Some of these relations have been already addressed by other authors following different perspectives $[9,10]$.

The above list is far from being exhaustive as other information related items could be taken into account. More work is needed on this subject.

Conflicts of Interest: The author declares no conflict of interest. 


\section{References}

1. Menant, C. Information and meaning. Entropy 2003, 5, 193-204. Available online: http://philpapers.org/rec/ MENIAM-2 (accessed on 18 July 2017).

2. Menant, C. Computation on Information, Meaning and Representations. Proposal for an Evolutionary Approach. In Information and Computation. Essays on Scientific and Philosophical Understanding of Foundations of Information and Computation; Dodig-Crnkovic, G., Burgin, M., Eds.; World Scientific: Singapore, 2011; pp. 255-286. Available online: https://philpapers.org/rec/MENCOI (accessed on 18 July 2017).

3. Menant, C. Turing Test, Chinese Room Argument, Symbol Grounding Problem. Meanings in Artificial Agents. In APA Newsletter Fall 2013, 2013. Available online: https://philpapers.org/rec/MENTTC-2 (accessed on 18 July 2017).

4. Menant, C. Proposal for an Evolutionary Nature of Self-Consciousness. 2014. Available online: https://philpapers.org/rec/MENPFA-3 (accessed on 18 July 2017).

5. Floridi, L. The Philosophy of Information; Oxford University Press: Oxford, UK, 2012.

6. Taddeo, M. Floridi, L. Solving the symbol grounding problem: A critical review of fifteen years of research. J. Exp. Theor. Artif. Intell. 2005, 17, 419-445. Available online: https://philpapers.org/rec/TADSTS (accessed on 18 July 2017).

7. Taddeo, M.; Floridi, L. A Praxical Solution of the Symbol Grounding Problem. Minds Mach. 2007, 17, 369-389.

8. Menant, C. Biosemiotics, Aboutness of Meaning and Bio-intentionality. Proposal for an Evolutionary Approach. In Proceedings of the Gatherings in Biosemiotics 2015, Copenhagen, Denmark, 30 June-4 July 2015. Available online: https://philpapers.org/rec/MENBAM-2 (accessed on 18 July 2017).

9. Burgin, M. Is information some kind of data? In Proceedings of the Third Conference on the Foundation of information Science (FIS 2005), Paris, France, 4-7 July 2005; pp. 1-31. Available online: http:// www.mdpi.net/fis2005/F.08.paper.pdf (accessed on 18 July 2017).

10. Dodig Crnkovic, G.; Hofkirchner, W. Floridi's open problems in philosophy of information, ten years later. Information 2011, 2, 327-359.

(c) 2017 by the author. Licensee MDPI, Basel, Switzerland. This article is an open access article distributed under the terms and conditions of the Creative Commons Attribution (CC BY) license (http://creativecommons.org/licenses/by/4.0/). 